\title{
2. DISCUSSION FOLLOWING VAN DE HULST'S REPORT
}

(Monday September 8, 1969)

\author{
Chairman: R. N. THомаS
}

Editor's remarks: This discussion on the opening day of the Symposium started in the afternoon and was consequently rather short. The version presented here is even shorter since some extensive remarks by Zel'dovich and Sunyaev have been transferred to a more proper location, the discussion following the Reports by Weaver and by Field (p. 77).

Pikel'ner: First, van de Hulst mentioned in passing the hypothetical concept of an Hil intercloud medium. I think this possibility can now be ruled out. Observations of the $21-\mathrm{cm}$ line show the existence of a hot, neutral gas between the clouds. Second, as for the heating of the gas, Faraday rotation and pulsar dispersion measurements show that the interstellar electron density is much higher now than was assumed 10 years ago. Therefore the cooling is much faster and cloud collisions cannot explain the high gas temperatures.

Van Woerden: Van de Hulst mentioned a kinetic energy density $W$ for the interstellar gas equal to $6 \times 10^{-12} \mathrm{erg} \mathrm{cm}^{-3}$. This number seems too large. In a volume of, say, $300 \mathrm{pc}$ diameter around the Sun, the average hydrogen density is $\left\langle n_{\mathrm{H}}\right\rangle=0.5 \mathrm{~cm}^{-3}$ and $\langle\varrho\rangle=1 \times 10^{-24} \mathrm{~g} \mathrm{~cm}^{-3}$ (helium included, no molecular hydrogen). With a velocity dispersion in one coordinate of $u=6 \mathrm{~km} \mathrm{sec}^{-1}$, I obtain $W=0.5 \times 10^{-12} \mathrm{erg} \mathrm{cm}^{-3}$. Inside clouds, hydrogen densities are much larger $\left(n_{\mathrm{H}} \approx 10 \mathrm{~cm}^{-3}\right)$, but internal velocities are much smaller, say, $u=2 \mathrm{~km} \mathrm{sec}^{-1}$; inside clouds, we therefore have $W=$ $1 \times 10^{-12} \mathrm{erg} \mathrm{cm}^{-3}$.

Pikel'ner: Much of space is filled with the intercloud medium, which has a low density. The value $W=6 \times 10^{-12} \mathrm{erg} \mathrm{cm}^{-3}$ refers to the cloud volume and cannot be compared with, for instance, the magnetic energy density, which fills all of space. In addition $I$ think that a proper density for standard clouds is 2 to 3 atom $\mathrm{cm}^{-3}$, instead of 10 atom $\mathrm{cm}^{-3}$.

Syrovat-skii: It seems to me that van de Hulst underestimated the energy supplied by supernovae. He gave $10^{-30} \mathrm{erg} \mathrm{cm}^{-3} \mathrm{sec}^{-1}$. But, if per supernova the energy release is about $10^{49} \mathrm{erg}$ or more and if there is one supernova each 30 years, then the energy supply for the galactic volume of $\lesssim 10^{67} \mathrm{~cm}^{3}$ is about $10^{-27} \mathrm{erg} \mathrm{cm}^{-3} \mathrm{sec}^{-1}$ or more.

Van de Hulst: My figure contains only that part of the energy that is indeed going into the cloud motion. Therefore my calculation contains an efficiency factor.

Woltjer: Kahn and I estimated this efficiency, using a rather extreme model (Kahn and Woltjer, 1967). My numbers indicate an energy per supernova outburst of a few times $10^{49} \mathrm{erg}$ and a conversion efficiency into interstellar cloud motion of about five 
per cent. [Kahn, F. D. and Woltjer, L.: 1967, IAU Symposium No. 31, Radio Astronomy and the Galactic System (ed. by H. van Woerden), Academic Press, New York, p. 117.]

Syrovat-skii: Then there is a real discrepancy. For one needs an efficiency factor of one per cent or less, and a supernova frequency of one per $300 \mathrm{yr}$ (ten times larger than is assumed at present) in order to obtain van de Hulst's estimate. In addition we have to neglect gas heating and expansion by supernova radiation and to neglect the action of supernova cosmic rays.

Shklovskii: I think van de Hulst's estimate is closer to reality than Syrovat-skii's. Shock waves will convert most of the energy of the explosion into thermal energy of the gas and transform it into UV and X-ray radiation. Most of the mechanical energy goes into X-ray emission.

Woltjer: The five per cent figure I gave is the fraction of the original mechanical energy that goes into interstellar motion. If originally there were more energy coming out as X-ray or UV radiation, then the total energy would go up, the efficiency would go down, but the input of kinetic energy would remain the same.

Colgate: I disagree with Shklovskii. Most of the energy ends up as kinetic energy as a result of adiabatic expansion. X-rays, gamma-rays, and optical light form only a small fraction of the total energy.

Menon: Dr. van de Hulst, you gave parameters for clouds of low temperature and density, but you did not discuss the possible range of their masses and whether or not there is any correlation between the densities and the masses. In particular, do the high density clouds have lower masses, and the low density clouds higher masses?

Van de Hulst: I cited mass values from van Woerden's review paper. Perhaps he can say more on this question. [Van Woerden, H.: 1967, IAU Symposium No. 31, Radio Astronomy and the Galactic System (ed. by H. van Woerden), Academic Press, New York, p. 3.]

Van Woerden: The data I assembled do not indicate a correlation such as Menon suggests. If anything, there is a slight positive correlation between density and mass.

Menon: The few cases where a high density has been observed are Hil regions. We know, from various mass estimates, that these objects have a fairly low mass. Therefore, the question is, do the low density clouds have large masses?

Van Woerden: The large masses I listed came from large clouds or cloud complexes where the density is not low (i.e., 10 to 50 atom $\mathrm{cm}^{-3}$ ). The total mass of a cloud depends to a large extent on how one defines the cloud. My summary did not include masses and densities of $\mathrm{H} I \mathrm{I}$ regions, and it contains no objects with densities larger than 100 atom $\mathrm{cm}^{-3}$.

Menon: To make an extreme statement: The only clouds with reliably known dimensions and densities are dense $\mathrm{H}$ II regions. For all other clouds we lack a suitable, accepted definition and the published values of the cloud parameters have been obtained by widely differing methods.

Van Woerden: I disagree with Menon in two ways. Among the high-density HiI regions, many are ionization bounded, not density bounded, so that the total mass of the cloud cannot be determined, but only the mass of the ionized part. On the other 
hand, as far as HI regions are concerned, I think there are obvious cases of wellbounded clouds.

Verschuur: I wish to raise the question of the term standard cloud. It seems to me a useless concept. For example, knowing what the average person in this room is like may tell us nothing about any individual member. Should we any longer use the term standard cloud? Does the term cloud still have some significance? I think we should observe specific regions only and discuss these, not the average ones.

Van de Hulst: I agree that the standard cloud is just a concept for limited use, for instance, in the context of components in a spectrum. We should always be very careful, first to define how we will use a model, and then to see whether or not the picture fits the observations.

Thomas: For the sake of mutual understanding I hope that at this Symposium we will give attention to questions of semantics as well as to the usual questions of physics. As an example the word 'cloud' was adopted historically to indicate that the interstellar medium was not homogeneous. The early question about this nonhomogeneous medium concerned how one obtained density fluctuations. One answer was that perhaps turbulence variations could produce them. But then one has to define what is meant by turbulence, which in turn means that one must define velocity fluctuations. I should like to ask: How do I describe the interstellar medium? Can I talk about an aerodynamic continuum? Can I talk about a density fluctuation and a velocity fluctuation? Does it make any sense to talk about clouds at all? Should we rather talk about density and velocity fields?

Weaver: In my Report I will show pictures of $21-\mathrm{cm}$ observations demonstrating the existence of interstellar clouds. But they are not what in the past has been called a standard cloud.

Spiegel: Could van de Hulst clarify his remarks on the distinction between velocities inside and velocities outside the clouds?

Van de Hulst: The question was: What is actually the difference between (i) a continuous velocity field and (ii) moving clouds with internal velocities? My point was that the observations themselves were really not quite sufficient to distinguish between these two possibilities. Historically it starts with the surprising results obtained by Adams that the interstellar lines could be separated into neat components. If you call each component a cloud, then you get a set of numbers representing 'internal' and 'external velocities'. But the more subtle question I now ask is whether or not such a simple interpretation is justified.

Field: I would suggest that a cloud is a mass of gas moving supersonically with respect to its own internal sound speed, but only subsonically with respect to the external sound speed. The external sound speed is much larger (ten times) than the internal sound speed because of a phase transition between a cold and a hot gas. This transition is not a shock wave, but rather a contact discontinuity across which there is pressure equilibrium. It is a critical point whether or not there can be lasting supersonic motions within this well-defined cloud. The observations suggest that the internal motions are in fact supersonic, because the observed widths of the lines exceed the 
sound speed by a factor of two or three. I wish to suggest a model in which cloud collisions maintain supersonic turbulence within individual clouds. This supersonic turbulence is caused by cloud collisions, which set the gas into motion on the largest scale within the cloud with the Mach number between 1 and 10. Zimmermann has shown how this process happens for central collisions. In the case of collisions offcenter, multiple shocks will form and will continue to cause supersonic motion for long periods. For example, rotation induced by the angular momentum of relative motion will result in a field of supersonic motion, with both rotational and compressive components. The compressive components will dissipate first, but the rotational ones may last longer and give the observed, mildly supersonic line widths. (Zimmermann, H.: 1968, Astron. Nachr. 290, 193, 211.)

Spiegel: It is very difficult to maintain supersonic turbulence inside a cloud and to keep the gas $\mathrm{HI}$, since it will heat up. Hence it seems unlikely that the observed velocity dispersions can be internal cloud turbulence.

Field: I agree with Spiegel that there is a problem. Perhaps it will take much longer to dissipate the rotational part than the compressive part of such turbulence. As the time between cloud collisions is only ten times the time of dissipation of compressive motions, perhaps the longer time to dissipate rotational motions will explain their presence in many clouds. Also, in the presence of a magnetic field cloud, collisions could generate large-amplitude Alfvén waves. These would add significantly to the observed widths. 